\title{
新型无机耐火纸
}

\author{
董丽颖，张永刚，朱英杰 \\ (中国科学院 上海硅酸盐研究所, 高性能陶瓷和超微结构国家重点实验室, 上海 200050)
}

摘 要: 新型无机耐火纸是以羟基磷灰石超长纳米线作为原料制备而成的, 具有优良的生物相容性、环境友好、物 理强度性能好、柔韧性高、耐高温、不燃烧、可用于书写和彩色打印, 有望用于档案等重要文件的长久保存。目前, 已制备出具有多种功能和用途的羟基磷灰石超长纳米线基新型纸张，包括抗菌耐火纸、防水耐火纸、苂光耐火纸、 耐高温标签纸、光(电)缆用阻燃耐火包带、快速检测试纸、生物医用纸、高效过滤纸等。新型无机耐火纸在特种纸、 吸附过滤、生物医学、隔热、耐高温、环境保护、能源等领域展现出广阔的应用前景。本文综述了最近几年来新 型羟基磷灰石超长纳米线基耐火纸研究取得的一些重要进展, 并且对该新型无机耐火纸未来的应用和产业化进行 了展望。

关 键 词: 羟基磷灰石; 纳米线; 耐火纸; 综述

中图分类号: TQ174 文献标识码: A

\section{A New Kind of Fire-resistant Inorganic Paper}

\section{DONG Li-Ying, ZHANG Yong-Gang, ZHU Ying-Jie}

(State Key Laboratory of High Performance Ceramics and Superfine Microstructure, Shanghai Institute of Ceramics, Chinese Academy of Sciences, Shanghai 200050, China)

Abstract: A new kind of highly flexible fire-resistant inorganic paper made from ultralong hydroxyapatite nanowires is reviewed. The fire-resistant inorganic paper exhibits high flexibility, high biocompatibility, nonflammability, high thermal stability, and excellent mechanical properties, which can be used for writing and printing, and is promising for permanent and safe storage of information, such as archives and important documents. The preparation process of this new kind of fire-resistant inorganic paper is environmentally friendly. The fire-resistant inorganic paper has many other potential applications in various fields. This new kind of fire-resistant inorganic paper provides a promising perspective for a possible substitute for the traditional cellulose paper, which has caused serious natural resource and environmental problems. In this review, we introduce the recent progress made in the research of this new kind of fire-resistant inorganic paper. Various kinds of fire-resistant paper sheets made from ultralong hydroxyapatite nanowires with different functions and applications are reviewed, including fire-resistant paper, superhydrophobic fire-resistant paper, antibacterial fire-resistant paper, photoluminescent fire-resistant paper, biomedical test paper, biopaper, refractory protection layer of the fiber-optic cable, and high-efficient filtration paper.

Key words: hydroxyapatite; nanowire; fireproof paper; review

收稿日期：2017-07-20; 收到修改稿日期：2017-10-25

基金项目：上海市科委基础研究重点项目(15JC1491001); 中国博士后科学基金(2016M591720); 国家自然科学基金青年基 金(51702342)

Key Basic Research Program of Science of and Technology Commission of Shanghai Municipality (15JC149001); China Postdoctoral Science Foundation (2016M591720); National Natural Science Foundation of China (51702342)

作者简介: 董丽颖(1985-), 女，博士. E-mail: lydong@mail.sic.ac.cn

通讯作者: 朱英杰, 研究员. E-mail: y.j.zhu@mail.sic.ac.cn 
纸是中国古代四大发明之一, 纸的发明结束了 古代简牍繁复的历史, 极大地促进了人类文化和科 学技术的传播与发展。如今, 纸已成为人类日常生 活和工作离不开的多用途产品, 即使是在科学技术 发展一日千里的当今电子信息时代, 纸仍然发挥着 重要的作用。纸的种类繁多, 其用途也越来越广泛, 不同用途的纸张对性能有不同的要求。

以植物纤维为原料的传统纸张需要消耗大量森 林资源, 传统造纸过程也会导致严重的环境污染。 以植物纤维原料抄造而成的纸张中含有较高比例的 木质素, 木质素的存在易导致纸张返黄。植物纤维 降解产生的酸性物质也易造成传统纸张长期保存后 强度性能下降。传统植物纤维纸的另一个致命弱点 是易燃性, 书籍和纸质文件在火灾中会被完全烧毁, 这也是众多纸质文物损毁消失的一个主要原因。传 统植物纤维纸既不防水、也不耐火, 这些缺点不仅 限制了传统纸的应用, 而且也为珍贵的纸质文物的 长久保存埋下了巨大隐患。例如, 2014 年 1 月, 法国 国家图书馆遭遇水灾, 超过一万册珍贵的书籍被水 淹而受到损毁 ${ }^{[1]}$ 。2 015 年 1 月 30 日, 莫斯科社会科 学信息研究所图书馆发生重大火灾, 大火持续了十 几个小时, 数以百万计的珍贵古籍图书资料在大火 中被烧毁; 更为雪上加霜的是在喷水灭火过程中又 使更多的图书资料受到破坏 ${ }^{[2]}$ 。

基于传统植物纤维纸面临的突出问题, 探索基 于无机材料的新型耐火纸就成为一个重要的研究课 题。但是, 无机耐火纸的发展往往受到无机材料自 身柔韧性差的制约。制造耐火纸的理想材料应同时 满足多个条件, 例如柔韧性高、不燃烧、耐高温、 白度高、无毒、可形成纤维、易于加工成薄层等。 然而, 很少有无机材料能够同时满足这些特性。

差基磷灰石 $\left(\mathrm{HAP}, \mathrm{Ca}_{10}\left(\mathrm{PO}_{4}\right)_{6}(\mathrm{OH})_{2}\right)$ 是一种天然 矿物质, 它也是脊椎动物骨骼和牙齿的主要无机成 份, 具有优良的生物相容性和生物活性, 并且环境 友好, 是一种理想的生物材料, 已经在生物医学领 域得到广泛应用, 例如药物输运、蛋白质吸附与释 放、骨骼及牙齿修复、生物成像、水处理等 ${ }^{[3-11]}$ 。 HAP 本身呈现优质的白色, 是制造纸的一种理想材 料。但是, HAP 材料脆性高、韧性低, 如何显著提高 HAP 材料的柔韧性是一个巨大挑战。

$\mathrm{Lu}$ 等 ${ }^{[12]}$ 发展了油酸钙前驱体溶剂热法, 成功 地制备出亲水性/疏水性可调控的 HAP 超长纳米线, 并发现采用 HAP 超长纳米线作为纸的构建材料可 以有效解决 HAP 材料高脆性、低韧性这一难题。该 团队以 HAP 超长纳米线作为构建材料, 采用简单的
真空抽滤技术制备出新型 HAP 超长纳米线耐火纸, 使“纸包得住火”成为现实。

\section{HAP 超长纳米线的合成}

HAP 材料的性能与应用受到其化学组成、结 构、形貌、尺寸以及结晶性等众多因素的影响, 因 此可以通过合成方法和制备条件来调控 HAP 材料 的性能, 并将其应用于各个不同领域中。到目前为 止, 人们已经成功合成出许多具有不同结构、形貌、 尺寸以及结晶性的 HAP 材料, 包括纳米颗粒、纳米 棒、纳米线、纳米片、三维自组装结构等 ${ }^{[13-21]}$ 。在 众多 HAP 材料中, 具有高长径比的 HAP 纳米线受 到广泛关注。HAP 纳米线比其它形貌的 HAP 纳米 材料具有更优良的力学性能和独特的生物学性能, 在力学增强、生物医学等领域具有良好的应用前景。 到目前为止, 国内外学者已经通过不同的方法成功 合成出 HAP 纳米线, 包括溶剂热法/水热法 ${ }^{[12,22-29] 、 ~}$ 微波辅助合成法 ${ }^{[30]}$ 、模板法 ${ }^{[31-32]}$ 、溶胶-凝胶法 ${ }^{[33]}$ 、 反胶束法 ${ }^{[34]}$ 等, 但是制备的 HAP 纳米线长度较短, 长度一般小于 $10 \mu \mathrm{m}$, 柔韧性较差, 不适合作为制 备高柔韧性新型无机耐火纸的原料。

$\mathrm{Lu}$ 等 ${ }^{[12]}$ 发展了油酸钙前驱体溶剂热法, 油酸 钙作为前驱体、以油酸、乙醇和水作为混合溶剂, 通 过溶剂热法成功合成出具有高柔韧性的 HAP 超长 纳米线。扫描电子显微镜(SEM)和透射电子显微镜 (TEM) 照片显示, 前驱体经溶剂热处理后得到的产 物为 HAP 超长纳米线(图 1(a) (c)), 且单根纳米线 呈单晶结构(图 1(c)插图)。制备的 HAP 超长纳米线 的直径约为 $10 \mathrm{~nm}$, 长度在几十微米到 $100 \mu \mathrm{m}$ 之 间。由于具有超长的长度和超高的长径比, 制备的 HAP 超长纳米线具有高柔韧性, 从图 1(a) (c)可以 看出, 许多 HAP 超长纳米线呈现自然弯曲状态(如 图 1(b)中箭头所示), 说明其具有高柔韧性, 可以作 为新型无机耐火纸的理想制备原料。将该 HAP 超长 纳米线分散在乙醇中, 用玻璃棒搅拌, 可形成很长 的纤维(如图 1(d) 所示, 长度约为 $28 \mathrm{~mm}$ ), 进一步证 明制备的 HAP 超长纳米线具有高柔韧性。制备的 HAP 超长纳米线具有高柔韧性主要是由于其超高 的长径比引起的。该油酸钙前驱体溶剂热法具有简 便、重复性好、具有较好的普适性等优点, 所以该 合成方法得到其他研究人员的关注 ${ }^{[25-26]}$ 。

另外, 制备的 HAP 超长纳米线还具有可调控的 亲疏水性能。文献中有关疏水性的 HAP 纳米材料的 制备和研究较为少见。本课题组发现, 通过改变实 验条件可以调节 HAP 超长纳米线的亲疏水性能, 并 

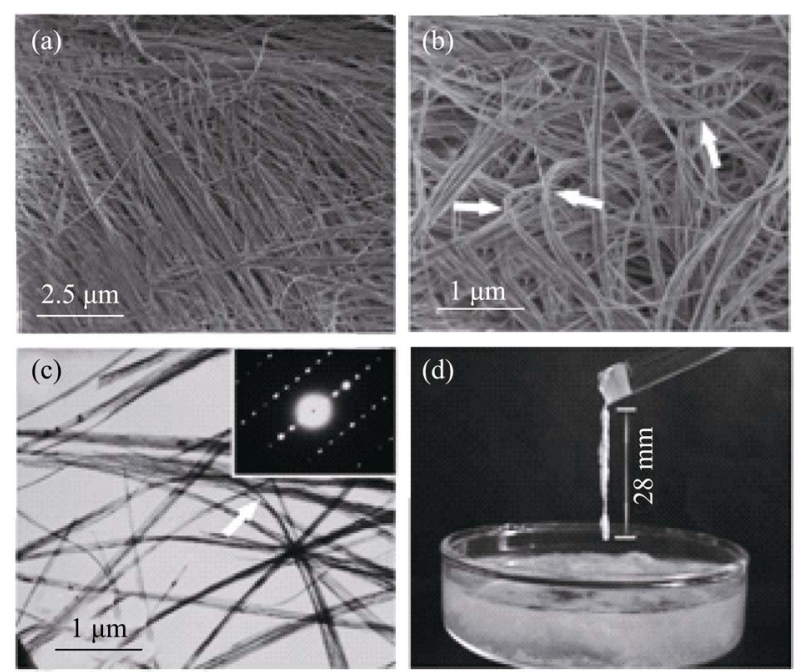

图 1 采用溶剂热法以油酸钙作为前驱体合成的高柔韧性 HAP 超长纳米线 ${ }^{[12]}$

Fig. 1 Highly flexible ultralong hydroxyapatite nanowires prepared by the calcium oleate precursor solvothermal meth$\operatorname{od}^{[12]}$

(a, b) SEM micrographs; (c) TEM micrograph with inset showing a SAED (selected-area electron diffraction) pattern of a single ultralong hydroxyapatite nanowire; (d) The formation of a long fiber with a length of $\sim 28 \mathrm{~mm}$ from an ethanol dispersion of ultralong hydroxyapatite nanowires

分别得到亲水性和疏水性的 HAP 超长纳米线。制备 的 HAP 纳米线压制成片, 测试其水接触角, 从而得 到其亲水性和疏水性。实验结果显示采用 $0.0926 \mathrm{~g}$ $\mathrm{NaH}_{2} \mathrm{PO}_{4} \cdot 2 \mathrm{H}_{2} \mathrm{O}$ 、溶剂热反应时间为 $5 \mathrm{~h}$ 制备样品的 接触角为 $137^{\circ}$, 具有高疏水性; 而当溶剂热反应时间 延长至 $23 \mathrm{~h}$ 时, 接触角缩小为 $108^{\circ}$ 。将 $\mathrm{NaH}_{2} \mathrm{PO}_{4} \cdot 2 \mathrm{H}_{2} \mathrm{O}$ 的用量增加至 $0.120 \mathrm{~g}$, 溶剂热反应时间为 5 和 $9 \mathrm{~h}$ 的样品接触角分别为 $95^{\circ}$ 和 $20^{\circ}$; 当溶剂热反应时间 延长至 $23 \mathrm{~h}$ 时, 样品则具有高亲水性, 导致水滴渗 入到样品中。产物的亲水性和疏水性可能与其表面 吸附的油酸分子或油酸根的数量有关 ${ }^{[10]}$ 。

在此基础上, Zhu 等 ${ }^{[28]}$ 陆续报道了 HAP 超长纳 米线的扩展合成方法, 例如, 采用溶剂热法和油酸 钙作为前驱体，在多种不同的一元醇中可制备得到 高长径比的 HAP 超长纳米线, 所用一元醇包括甲 醇、乙醇、正丙醇、异丙醇、丁醇、戊醇和己醇等, 制备的 HAP 超长纳米线的长度可达到 $100 \mu \mathrm{m}$ 以上, 通过优化制备条件, HAP 纳米线的长度甚至可以达 到接近 $1000 \mu \mathrm{m}$, 而且在大多数情况下产物是由多 个纳米线沿同一方向自组装在一起形成的束状结 构 ${ }^{[27]}$ 。另一个研究工作是采用溶剂热法, 以油酸钙 为前驱体, 利用一系列不同的磷酸盐作为磷源, 成 功合成出 $\mathrm{HAP}$ 超长纳米线, 所用磷源包括 $\mathrm{Na}_{2} \mathrm{HPO}_{4}$ 、 $\mathrm{NaH}_{2} \mathrm{PO}_{4} 、 \mathrm{Na}_{3} \mathrm{PO}_{4} 、 \mathrm{Na}_{5} \mathrm{P}_{3} \mathrm{O}_{10}$ 和 $\mathrm{Na}_{4} \mathrm{P}_{2} \mathrm{O}_{7}$ 等, 制备的
HAP 超长纳米线的长度可达到 $100 \mu \mathrm{m}$ 以上, 而且 在大多数情况下产物是由多个纳米线沿同一方向自 组装在一起形成的束状结构。

最近, Li 等 ${ }^{[29]}$ 针对油酸-醇-水混合反应体系列 出了合成 HAP 超长纳米线存在的缺点, 例如成本较 高、产物浆料偏黄、产物清洗繁琐等问题, 进一步 发展了 HAP 超长纳米线的新制备方法, 利用油酸钠 代替油酸, 去除了有机溶剂油酸和醇, 并采用环境 友好的水作为唯一溶剂, 成功地制备出网状结构 HAP 超长纳米线, 产物浆料白度高, 该制备方法可 大大降低制备成本, 并且环境友好。Zhu 等在 HAP 超长纳米线的放大制备技术探索方面也取得了良好 研究进展，发展了体积为 $10 \mathrm{~L}$ 级反应釜的制备技术， 并且具有良好的可重复性 ${ }^{[29,35]}$ 。

\section{2 新型 HAP 超长纳米线无机耐火纸}

2014 年, $\mathrm{Lu}$ 等 ${ }^{[12]}$ 报道了油酸钙前驱体溶剂热法, 采用该方法成功地制备出 HAP 超长纳米线, 并以 $\mathrm{HAP}$ 超长纳米线作为原料成功地制备出新型 HAP 超长纳米线耐火纸(图 2(a))。而采用 HAP 短纳米棒 或球形颗粒则不能通过同样的方法制备出高柔韧性 耐火纸，说明纳米线的长度对耐火纸的柔韧性具有 很大的影响。耐火纸的尺寸和厚度可以通过改变过 滤漏斗的尺寸和 HAP 超长纳米线的用量来调控。制 备的新型无机耐火纸具有高柔韧性，可以任意卷曲， 可耐高温，不燃烧，并且具有优良的书写和打印功

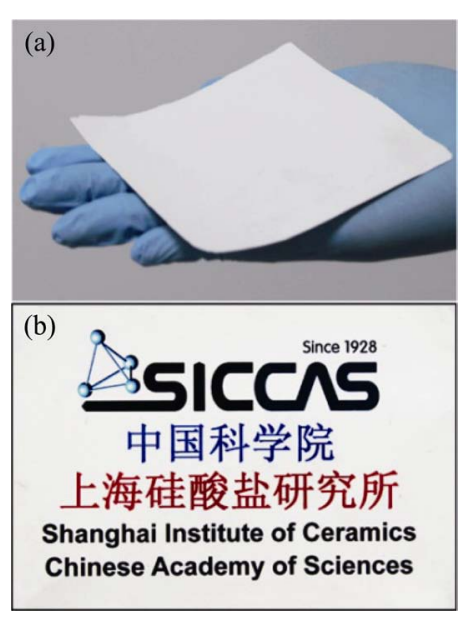

图 2 (a) 新型 HAP 超长纳米线耐火纸的数码照片和(b) 用 喷墨打印机在 HAP 超长纳米线耐火纸上打印的不同颜色图 案和文字的数码照片 ${ }^{[12]}$

Fig. 2 (a) A digital image of the new kind of highly flexible fire-resistant paper made from ultralong hydroxyapatite nanowires; (b) English words and Chinese characters with different colors printed on the fire-resistant ultralong hydroxyapatite nanowire paper by using a commercial ink-jet printer ${ }^{[12]}$ 
能, 利用普通喷墨打印机可以将不同颜色的图案和 文字清晰地打印在耐火纸上, 如图 2(b)所示; 该新 型耐火纸有望应用于书籍、重要文件及档案的长久 安全保存 ${ }^{[10]}$ 。

通过大量的实验探索, $\mathrm{Li}$ 等 $^{[29]}$ 发展了 HAP 超长 纳米线浆料的优化配方、无机胶粘剂优化配方和耐 火纸抄造技术，制备的 HAP 超长纳米线耐火纸的强 度等性能得到明显提高, 耐火纸的尺寸由原来的几 厘米放大到 $\mathrm{A} 4$ 尺寸 $(21 \mathrm{~cm} \times 29.7 \mathrm{~cm})$, 可直接用于 打印机打印, 效果良好(图 3)。目前, 研发的 HAP 超 长纳米线耐火纸的主要性能指标可达到复印纸国 家标准, 可应用于书写、复印、打印等特种办公用 纸、耐高温标签纸以及档案等重要文件的长久安全 保存等。

制备的新型 HAP 超长纳米线耐火纸具有良 好的耐火性能和高热稳定性。当用酒精灯加热时, 普通的复印纸迅速燃烧, 仅几秒钟就化为灰烬 (图 4(a) (c)); 而新型无机耐火纸即使在酒精灯上 加热 $5 \mathrm{~min}$ 后仍完好无损, 而且采用耐高温石墨书 写的字体也保存完好(图 4(d) (f))。此外, 将 HAP 超 长纳米线耐火纸在 $450^{\circ} \mathrm{C}$ 电炉中加热 $1 \mathrm{~h}$, 耐火纸及 在其上面书写的字体仍能完好保持, 表明该耐火纸 具有高热稳定性(图 4(g) (i) $)^{[12]}$ 。

\section{3 新型 HAP 超长纳米线耐火纸的功 能化及其应用}

\section{1 高效吸附耐火纸}

近年来, 原油、石油产品以及其它有机溶剂泄 漏的事件时有发生, 造成了严重的环境污染。因此,

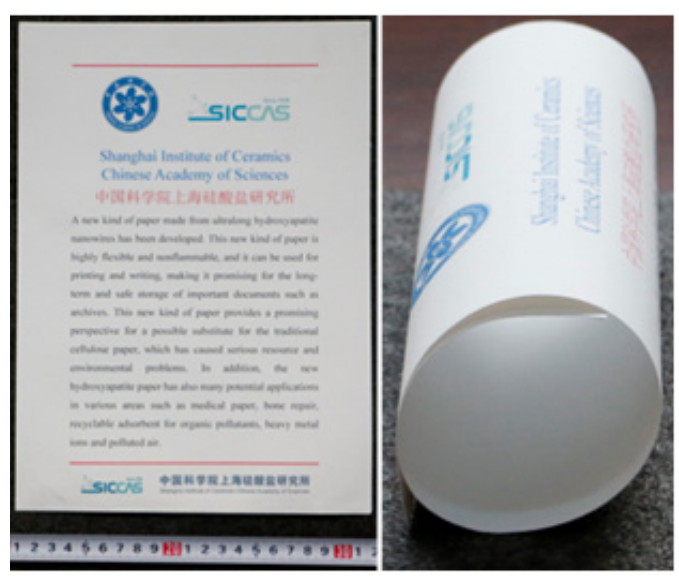

图 3 制备的 $\mathrm{A} 4$ 尺寸 $(21 \mathrm{~cm} \times 29.7 \mathrm{~cm})$ 的 HAP 超长纳米线耐 火纸的数码照片 ${ }^{[29]}$

Fig. 3 Digital images of the highly flexible fire-resistant ultralong hydroxyapatite nanowire paper with an $\mathrm{A} 4 \operatorname{size}^{[29]}$

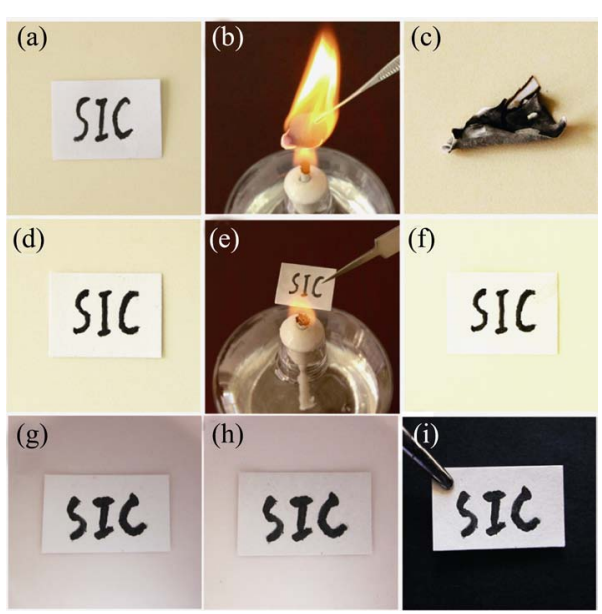

图 4 新型高柔韧性 HAP 超长纳米线耐火纸的耐火性能、 热稳定性及其与普通复印纸的对比实验 ${ }^{[12]}$

Fig. 4 Illustration of the excellent fire-resistance and hightemperature resistance of the as-prepared highly flexible fireresistant paper based on ultralong hydroxyapatite nanowires, and the commercial cellulose paper is used for comparison ${ }^{[12]}$

采用疏水性材料处理油污引起了人们的兴趣。但是, 通常情况下这些材料对有机污染物的吸附率低、循 环利用性能差、材料本身对环境造成污染，使其不 能得到广泛应用，人们期望通过发展新的吸附材料 来解决这些问题。

HAP 材料具有优良的生物相容性，且环境友好, 但目前有关 HAP 纳米材料在有机污染物处理方面 的应用研究却报道较少。 $\mathrm{Lu}$ 等 ${ }^{[12]}$ 研究了新型 HAP 超长纳米线耐火纸作为吸附材料对几种有机物的吸 附性能。实验结果表明, 耐火纸对多种有机污染物 具有较高的吸附量(图(5)), 例如对氯仿的吸附量可 达到 7.3 g/g (图 5(a)), 对多种有机污染物也有较高 的吸附量。采用耐火纸作为填充物制备出一种简单 的过滤柱, 可用于处理有机溶剂和水的混合物。如 图 5(c)所示, 将甲苯 (用油红 $O$ 染色)与水的混合物 加入到过滤柱后, 水通过了过滤柱, 而甲苯则被耐 火纸吸附保留在过滤柱中。该装置借助 HAP 超长纳 米线耐火纸对有机溶剂的高效吸附能力, 可将甲苯 和水有效分离，使水得到净化。

对吸附剂的回收重复利用可大幅减少材料的消 耗, 并节约成本。将吸附了有机溶剂的 HAP 超长纳 米线耐火纸置于一种简单的有机溶剂蒸馏装置中并 加热处理, 可有效分离并收集有机溶剂, 耐火纸也 可以得到回收利用。回收后的耐火纸几乎没有残留 的有机溶剂, 而耐火纸本身也无明显损坏。采用回收 后的耐火纸再次吸附有机溶剂, 即使循环使用 5 次, 其吸附效率也没有明显降低(图 5(b))。而对于常见的 有机高分子吸附剂, 由于自身热稳定性差而不能 

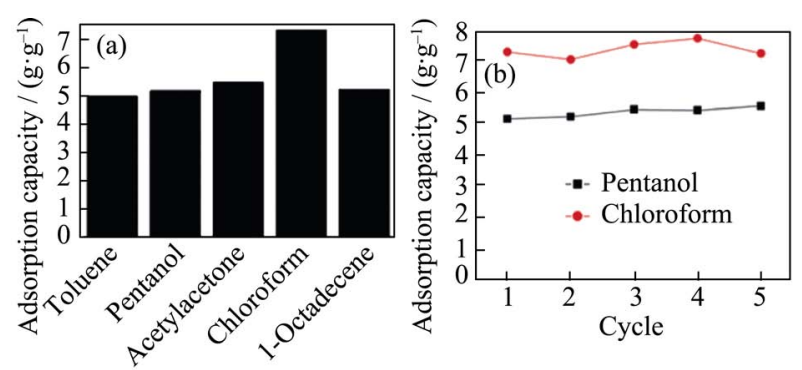

(c)

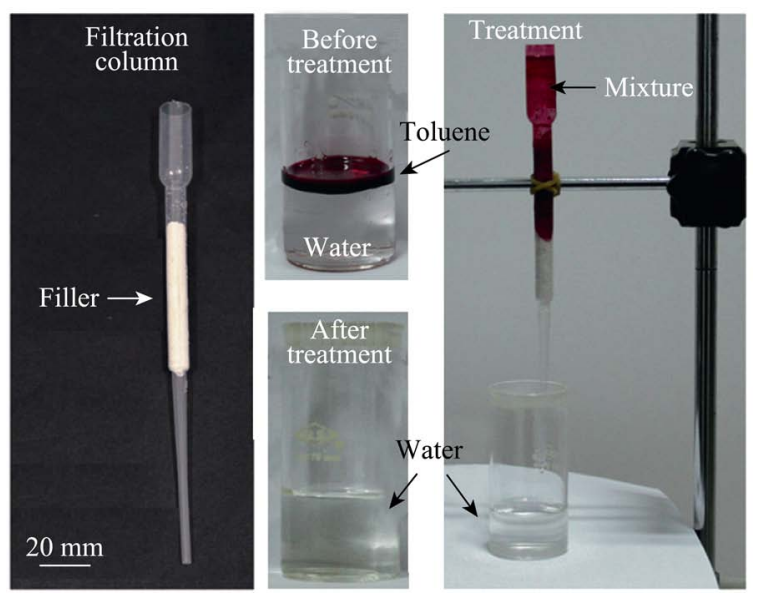

图 5 HAP 超长纳米线耐火纸对有机污染物的吸附性能 ${ }^{[12]}$

Fig. 5 Adsorption properties of the highly flexible fire-resistant paper made from ultralong hydroxyapatite nanowires ${ }^{[12]}$

用加热方法回收, 只能通过萃取的方法处理, 但是 萃取法很难将有机溶剂完全去除, 并且会对环境造 成污染。HAP 超长纳米线耐火纸具有优良的热稳定 性和耐火性能, 环境友好, 可循环使用, 是一种具 有良好应用前景的有机污染物可再生高效吸附剂, 在污水处理和环境保护领域具有良好的应用前景 ${ }^{[12]}$ 。

\section{2 防水耐火纸}

超疏水材料具有抗污、防雾、自清洁等优点, 在 多个领域具有良好的应用前景 ${ }^{[36-37]}$ 。虽然学术界对 于超疏水材料的制备已经进行了深入的研究, 但是 仍难于获得稳定、可抗机械损伤和外界严酷环境的 超疏水性能。超疏水表面遭受诸如刮擦、磨损等物 理破坏或处于高温等严酷环境中往往不能很好地保 持超疏水状态。目前, 制备高稳定性的超疏水材料 仍然是一个很大的挑战。

针对这一难题, Chen 等 ${ }^{[38]}$ 提出了层状结构超疏 水耐火纸的概念, 采用经表面修饰的 HAP 超长纳米 线作为原料制备出具有层状结构的新型防水耐火纸, 该防水耐火纸不仅表面层呈现超疏水状态，而且其 内部也呈现超疏水状态, 当表面层受到破坏后, 内 部暴露出来的新层仍然保持超疏水状态, 从而可实 现耐火纸超疏水性能的高稳定性，在耐火纸受到机 械损伤(例如手指摩擦、胶带粘贴剥离、砂纸磨损、 刀划割等)或在高温严酷环境中仍能保持防水性能,
并可同时实现防水和隔热耐火等多功能性。制备的 防水耐火纸不仅具有优良的超疏水性能, 对多种商 业饮料如矿泉水、橙汁、红茶、牛奶和咖啡等也具 有良好的超疏水性能(图 6)。另外，制备的防水耐火 纸还具有良好的自清洁功能, 水会形成球形水珠在 纸的表面自由地滚动并带走灰尘等污物而实现自 清洁。如果防水耐火纸用于露天广告牌等, 利用雨 水可自动保持清洁而免去人工清洗的麻烦, 并降低 成本。另外，防水耐火纸具有超疏水性能的同时， 还呈现超亲油状态，可应用于油和水的快速高效分 离(图 7) ${ }^{[38]}$ 。防水耐火纸还具有优良的隔热耐火性 能，可应用于保护易燃物品免于火灾焚毁。此外， 由无机纳米线自组装制备的层状结构耐火纸可以 实现调控其层间距和孔径, 有望应用于控制水输运 的阀门 ${ }^{[39]}$ 。

\section{3 抗菌耐火纸}

纸和纸制品是人们工作和生活中不可缺少的用 品, 一些使用和交换频繁的纸张, 例如病历纸、钞票 纸和各种票据等, 细菌容易附着于纸的表面并通过 人群传播, 对人体健康造成威胁。另外, 重要馆藏书 籍和文物的保存也需要预防霉菌。因此, 研制新型 抗菌无机耐火纸具有重要的研究意义和实用价值。 最近, Xiong 等 ${ }^{[40]}$ 发展了新型 HAP 超长纳米线抗菌 耐火纸的制备技术，通过一步溶剂热法制备出分散 性好、含量可调控的纳米银复合 HAP 超长纳米线, 并成功制备出尺寸和厚度可调控的新型 HAP 超长 纳米线抗菌耐火纸，该纸具有高柔韧性、良好的生 物相容性和高效抗菌性能, 并且耐高温不燃烧。该 抗菌耐火纸对金黄色葡萄球菌和大肠杆菌均具有高 效和长效的抗菌活性, 同时随着银纳米颗粒含量的 增加, 抗菌效率提高。另外, Xiong 等 ${ }^{[41]}$ 还研发出一 种 HAP 超长纳米线双模式抗菌纸, 在 HAP 超长纳 米线耐火纸中装载二种抗菌剂, 即银纳米颗粒和抗 生素(环丙沙星)。新型双模式抗菌纸具有高抗菌剂 装载量、协同高效抗菌性能和长效抗菌活性, 并且 具有优良的循环利用效果。

\subsection{HAP 超长纳米线耐火纸应用于光(电)缆} 阻燃耐火包带

随着通讯技术的发展，光(电)缆被广泛应用于 各个领域。火灾发生时, 保持一段时间的通讯畅通 对于减少人员和财产损失至关重要。因此，防火光 (电)缆在当今信息社会各领域具有至关重要的地 位。现有的光(电)缆阻燃包带主要有以下几种: 双面 合成云母带、金云母带、陶瓷化聚烯烃带和玻璃纤 维带等, 这几种阻燃包带虽然具有一定的阻燃性能, 

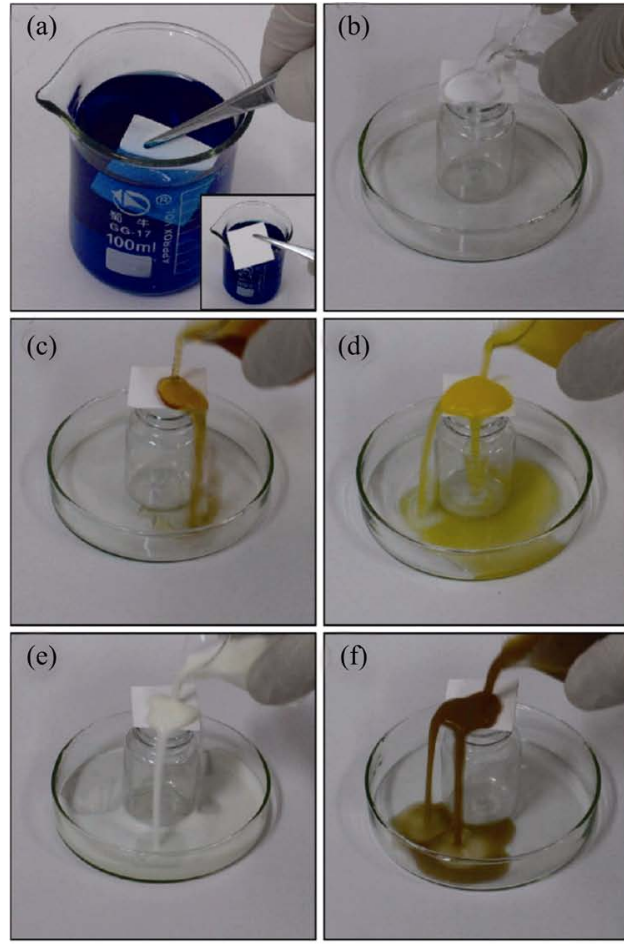

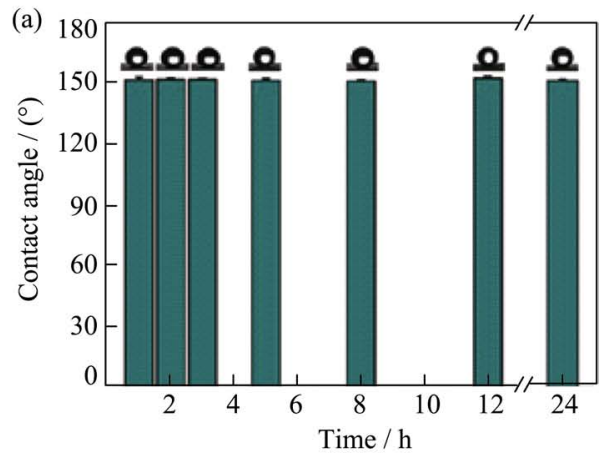

(b)

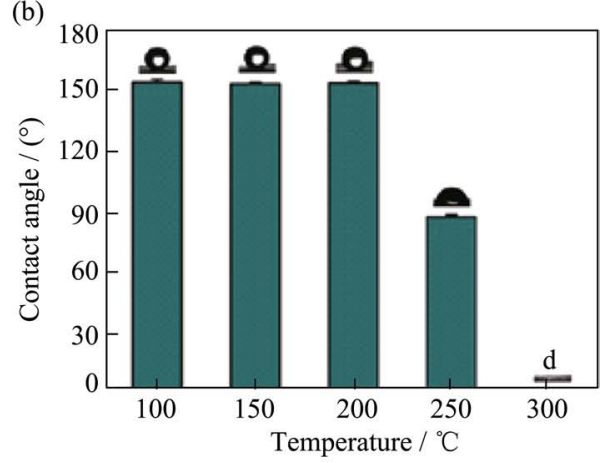

图 6 新型 HAP 超长纳米线防水耐火纸对水和多种商业饮料具有优良的超疏水性能和优异的热稳定性 ${ }^{[38]}$

Fig. 6 Liquid repellency tests for a new kind of fire-resistant superhydrophobic layered paper based on ultralong hydroxyapatite nanowires and its excellent thermal stability ${ }^{[38]}$
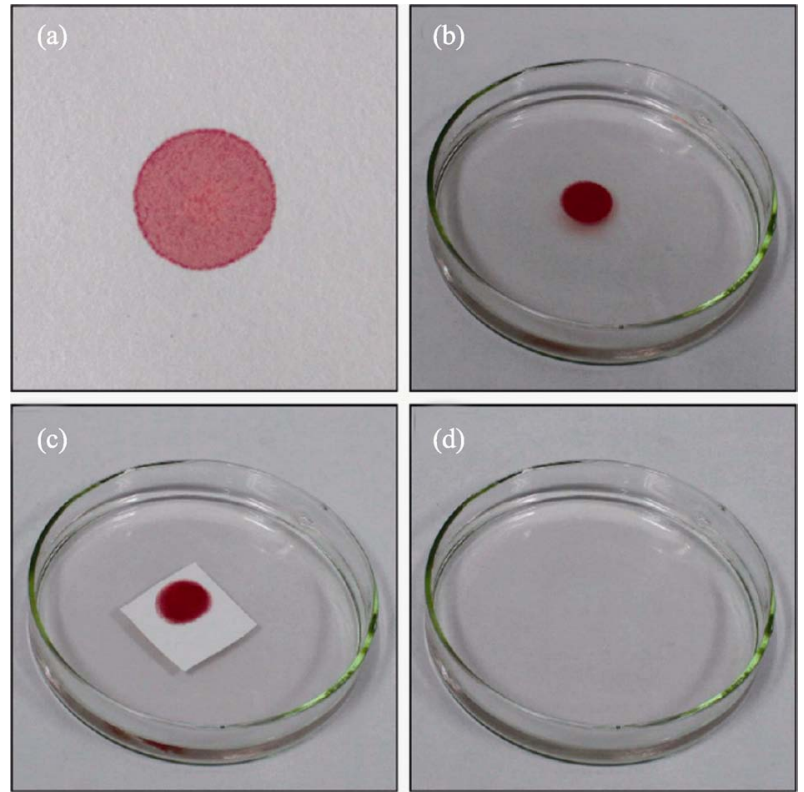

图 7 新型 $\mathrm{HAP}$ 超长纳米线防水耐火纸可应用于油和水的 高效快速分离 ${ }^{[38]}$

Fig. 7 Oil/water separation of the fire-resistant superhydrophobic ultralong hydroxyapatite nanowire paper ${ }^{[38]}$

但其隔热性能不理想，火焰灼烧后易脆化，一些包 带火焰灼烧时会释放出烟雾和有毒有害气体，对在 场人员造成伤害。

Dong 等 ${ }^{[42]}$ 针对现用光(电)缆阻燃包带存在的 问题, 利用自制的网状结构 HAP 超长纳米线作为主
体材料，以玻璃纤维作为“骨架”材料，加入一定量 的无机胶粘剂，制备出一种具有类似“钢筋混凝土” 高强度整体结构的新型耐火纸。将该耐火纸用作光 (电)缆阻燃耐火包带, 具有低的导热系数和优异的 隔热和耐火性能, 并且环境友好、在火灾中无有毒 有害气体产生。与商品化阻燃包带相比, 火焰灼烧 时耐火纸可使内部温度降低大约 $150^{\circ} \mathrm{C}$ 以上, 高温 灼烧后耐火纸完好无损, 这有利于火灾中保护光 (电)缆内部缆芯。新型无机耐火纸阻燃耐火包带在 防火光(电)缆领域具有良好的应用前景。

\subsection{HAP 超长纳米线快速检测试纸}

近年来, 快速分析检测技术在化学检测、医学 诊断、司法鉴定、环境监测和食品检测等领域具有 广泛的应用。仪器分析方法具有高测定精度和低检 出限, 但一般使用的是大型精密仪器, 且采用交流 电做电源, 操作较为复杂, 使用不方便, 不适合现 场快速检测。随着科学技术的进步, 各种快速高效 的现场分析检测手段相继出现。试纸法作为一种快 速的现场检测方法, 其特点是操作简单、携带方便、 价格便宜, 并具有一定的选择性、准确性和灵敏度, 在医疗卫生、食品、水质、空气及其它检测方面具 有广泛应用。然而, 天然酶价格高, 其制备、提纯和 储存均耗时耗力, 而且检测活性易受外界环境如 $\mathrm{pH}$ 、温度等影响。 
近年来, 具有天然酶活性的人工模拟酶受到人 们的广泛关注。通过化学方法合成的人工模拟酶成 本低, 催化活性较稳定, 有望取代部分天然酶应用 于分析检测领域。Chen 等 ${ }^{[43]}$ 发明了一种 HAP 超长 纳米线基快速检测试纸，该方法在 HAP 超长纳米线 上原位生长具有类过氧化物酶活性的 $\mathrm{Fe}$ 基金属有 机框架复合物, 利用 HAP 超长纳米线上的钙离子与 金属有机框架复合物上的羧基之间的耦合作用，制 备具有核壳结构的 HAP 超长纳米线@金属有机框 架复合物纳米纤维, 并将其用于制备快速检测试 纸。该检测试纸本身具有类似过氧化物酶的活性, 可用于葡萄糖、过氧化氢等物质的快速检测。该检 测试纸制备简单, 成本较低, 稳定性好, 可实现多 次重复回收利用, 只需将使用后变色的检测试纸 浸泡在酒精中 $30 \mathrm{~min}$, 检测试纸就重新变回原来 的颜色。

\subsection{HAP 超长纳米线基生物纸}

由单一 HAP 组成的材料通常脆性高, 柔韧性差, 难以塑造成各种生物医学应用所需的特定形状。此 外, 在一些特定的生物医学应用中需要使用柔性生 物材料。为此, 设计合成具有良好柔韧性和优异力 学性能的 HAP 与生物高分子的复合材料具有重要 的研究价值。壳聚糖是一种来源丰富的天然高分子 材料, 具有良好的生物相容性、可降解性和抗菌性 能，可用作止血剂和皮肤伤口敷料以促进血液凝固 和伤口愈合。胶原蛋白是生物体中重要的有机成分, 具有优异的生物相容性和生物降解性, 是一种优良 的生物材料。因此, 将 HAP 超长纳米线与生物高分 子壳聚糖或胶原蛋白复合有望制备出性能优异的柔 性生物材料。

最近, Sun 等 ${ }^{[44-45]}$ 研制出具有良好柔韧性和优 异力学性能的新型 HAP 超长纳米线基生物纸。该生 物纸以 HAP 超长纳米线作为构建材料, 与具有良好
生物相容性的天然生物高分子例如壳聚糖或胶原蛋 白复合制备而成。制备的生物纸具有诸多优点，例 如柔韧性好、生物相容性和生物活性高、力学性能 优异、HAP 超长纳米线的含量可在 $0 \sim 100 \mathrm{wt} \%$ 大范 围内连续调控(图 8), 可解决传统生物膜 HAP 含量 偏低且力学性能差等难题。通过改变 HAP 超长纳米 线的含量可调控生物纸的表面润湿性、溶胀率、水 蒸气透过速率以及力学性能。该生物纸是一种性能 优异的新型柔性生物材料, 有望应用于生物医学领 域，例如皮肤创伤修复、骨裂或骨折包扎固定、骨 缺损修复、各种用途的医用纸等。

\section{7 多模式防伪耐火纸}

荧光防伪纤维是目前常用的防伪材料之一, 具 有使用方便、易辨别的优点, 广泛应用于钞票、有 价证券、防伪证件、防伪包装材料等。荧光防伪纤 维的制备是通过物理或化学方法将荧光物质引入到 有机高分子基质中。许多有机高分子荧光纤维制备 工艺复杂, 且难以回收利用, 在自然环境中难降解, 易造成环境污染; 另外, 苂光物质易从有机高分子 基质中溶出，苂光稳定性较差，受到光照、细菌等外 界条件影响易老化，并且高温条件下易碳化或燃烧。

采用稀土离子掺杂的方法可以对羟基磷灰石纳 米材料进行功能化 ${ }^{[7,46-50]}$ 。Yang 等 ${ }^{[51]}$ 通过原位掺杂 的方法在 HAP 超长纳米线中引入不同的稀土离子, 发光颜色和发光强度可以通过掺杂稀土离子的种类 和浓度进行调控，实现了 $\mathrm{HAP}$ 超长纳米线的苂光功 能化, 并以此为原料构建了 HAP 超长纳米线荧光耐 火纸。图 9 显示普通植物纤维纸在 $300^{\circ} \mathrm{C}$ 热处理 10 min 即碳化变黑; 而 $\mathrm{Tb}^{3+}$ 掺杂 $\mathrm{HAP}$ 超长纳米线 苂光耐火纸在 $300^{\circ} \mathrm{C}$ 热处理 $60 \mathrm{~min}$ 外观并没有明 显变化, 可以很好地保持白度, 热处理后在紫外光 ( 365 nm) 照射下其发光性能没有明显下降。进一步 通过表面修饰的方法, 在 HAP 荧光超长纳米线表
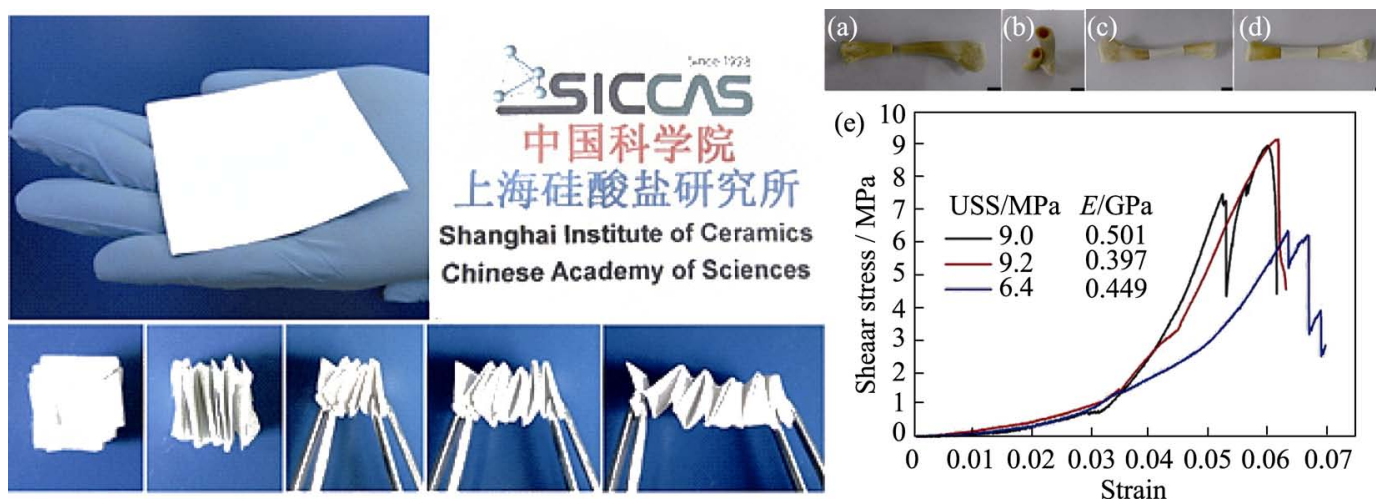

图 8 制备的 $90 \mathrm{wt} \%$ HAP 超长纳米线/壳聚糖复合生物纸的数码照片和力学性能 ${ }^{[44]}$

Fig. 8 Digital images and the shear stress-strain curves of the as-prepared $90 \mathrm{wt} \%$ ultralong hydroxyapatite nanowire/chitosan biopaper ${ }^{[44]}$ 

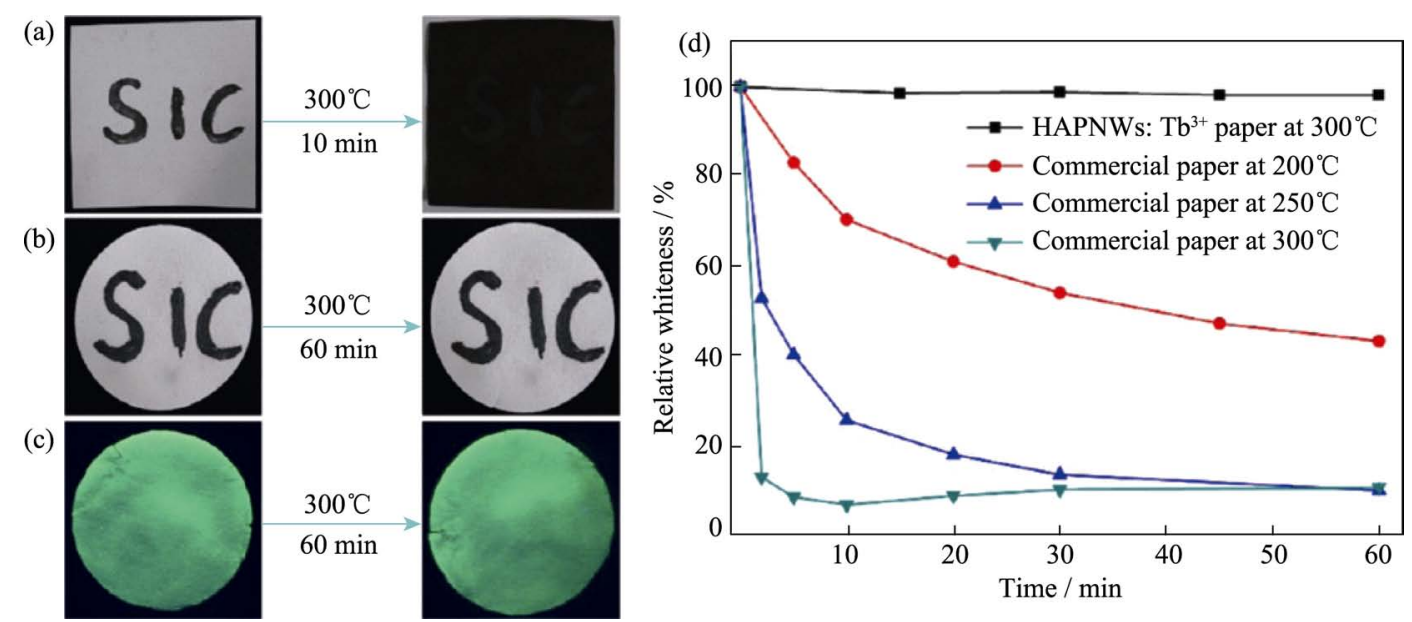

图 9 普通植物纤维纸(a)和 $\mathrm{Tb}^{3+}$ 掺杂 $\mathrm{HAP}$ 超长纳米线荧光耐火纸(b)在热处理前后的数码照片; (c) 制备的 $\mathrm{Tb}^{3+}$ 掺杂 $\mathrm{HAP}$ 超 长纳米线荧光耐火纸 $300^{\circ} \mathrm{C}$ 处理 $60 \mathrm{~min}$ 后在紫外光 $(\sim 365 \mathrm{~nm})$ 照射下发光性能保持良好; (d) 制备的 $\mathrm{Tb}^{3+}$ 掺杂 $\mathrm{HAP}$ 超长纳米线 苂光耐火纸和普通植物纤维纸在热处理前后的白度变化 ${ }^{[51]}$

Fig. 9 Digital images of two kind of paper sheets before and after thermal treatment

(a) Common commercial paper made of plant cellulose fibers; (b) $\mathrm{Tb}^{3+}$-doped HAP ultralong nanowire photoluminescent fire-resistant paper; (c) $\mathrm{Tb}^{3+}$-doped HAP ultralong nanowire paper under UV irradiation $(\sim 365 \mathrm{~nm})$; (d) Whiteness change of the common commercial paper and the $\mathrm{Tb}^{3+}$-doped HAP ultralong nanowire paper after thermal treatment at different temperatures ${ }^{[51]}$

面修饰油酸钠，实现了 HAP 超长纳米线苂光耐火纸 的超疏水功能化。在以上研究基础上，采用多功能 化的 HAP 超长纳米线作为原料, 成功地构建了 HAP 超长纳米线基多模式防伪耐火纸，在产品标 签、防伪印钞、防伪证件、高温环境下的苂光指示、 耐高温柔性显示器件等领域具有良好的应用前景 ${ }^{[51]}$ 。

\section{8 新型 HAP 超长纳米线过滤纸}

近年来, 雾霧天气频繁出现, 严重的空气污染 问题备受人们关注。空气中 $\mathrm{PM}_{2.5}$ 细颗粒物严重威 胁人们的身体健康。 $\mathrm{PM}_{2.5}$ 细颗粒物是指环境空气动 力学直径小于或等于 $2.5 \mu \mathrm{m}$ 的颗粒物, 其在空气 中的含量越高, 空气污染就越严重。 $\mathrm{PM}_{2.5}$ 细颗粒物 的粒径小, 活性高, 且在大气中停留时间长、输送距 离远, 影响范围大, 被吸入人体后可进入支气管甚 至肺部, 引发多种疾病; 此外, $\mathrm{PM}_{2.5}$ 细颗粒物可作 为有毒有害物质、病毒和细菌的载体对疾病的传播 推波助澜 ${ }^{[52-53]}$ 。因此, 研制空气 $\mathrm{PM}_{2.5}$ 细颗粒物的高 效过滤材料具有重要的意义。

Xiong 等 ${ }^{[54]}$ 成功研制出一种可高效清除空气 $\mathrm{PM}_{2.5}$ 细颗粒物的新型羟基磷灰石超长纳米线基过 滤纸及以其作为滤芯的过滤口罩。该新型过滤纸可 有效拦截、吸附和过滤空气 $\mathrm{PM}_{2.5}$ 细颗粒物, 过滤效 率高。另外, 为了显著改善新型过滤纸的透气性, 该 团队采用羟基磷灰石超长纳米线作为主要构建材料, 与植物纤维复合, 从而形成多级复合孔道结构, 实 现了在保持空气 $\mathrm{PM}_{2.5}$ 细颗粒物高过滤效率的同时, 大大提高了过滤纸的透气性。新型过滤纸对空气
$\mathrm{PM}_{2.5}$ 和 $\mathrm{PM}_{10}$ 颗粒物的过滤效率均高于 $95 \%$, 并且 具有良好的透气性, 过滤效率优于多种商品化口 罩。更重要的是, 该新型过滤纸在中度、重度、严 重污染等不同程度的空气污染环境中, 对 $\mathrm{PM}_{2.5}$ 细 颗粒物的过滤效率均高于 95\%，可作为核心过滤材 料嵌入日常过滤口罩中, 实现在各种雾霧天气环境 中个人的有效防护。另外该新型过滤纸还可多次重 复和长时间使用(图 10)。此外, 该团队还研制出一 种具有抗菌功能的新型羟基磷灰石超长纳米线基过 滤纸，对大肠杆菌和金黄色葡萄球菌等细菌具有高 效抗菌活性，有望应用于高效抗菌过滤口罩。该新 型过滤纸还有望作为高效滤芯材料应用于空气净化 器、空调等产品中。

\section{4 总结与展望}

HAP 是一种天然矿物质, 也是脊椎动物骨骼和 牙齿的主要无机成份, 具有优良的生物相容性和生 物活性, 耐高温耐火, 并且环境友好, 本身呈现优 质的白色, HAP 超长纳米线具有高柔韧性, 这些优 点使 HAP 超长纳米线成为一种理想的耐火纸制造 原料。采用 HAP 超长纳米线作为构建材料, 可制备 出新型 HAP 超长纳米线耐火纸, 使 “纸包得住火” 成为现实。新型 HAP 超长纳米线耐火纸具有多个优 点, 例如, 柔韧性高、白度高(不需要漂白)、生物相 容性好、环境友好、耐高温、不燃烧、隔热性能好、 可用于书写打印等。该新型无机耐火纸有望应用于 

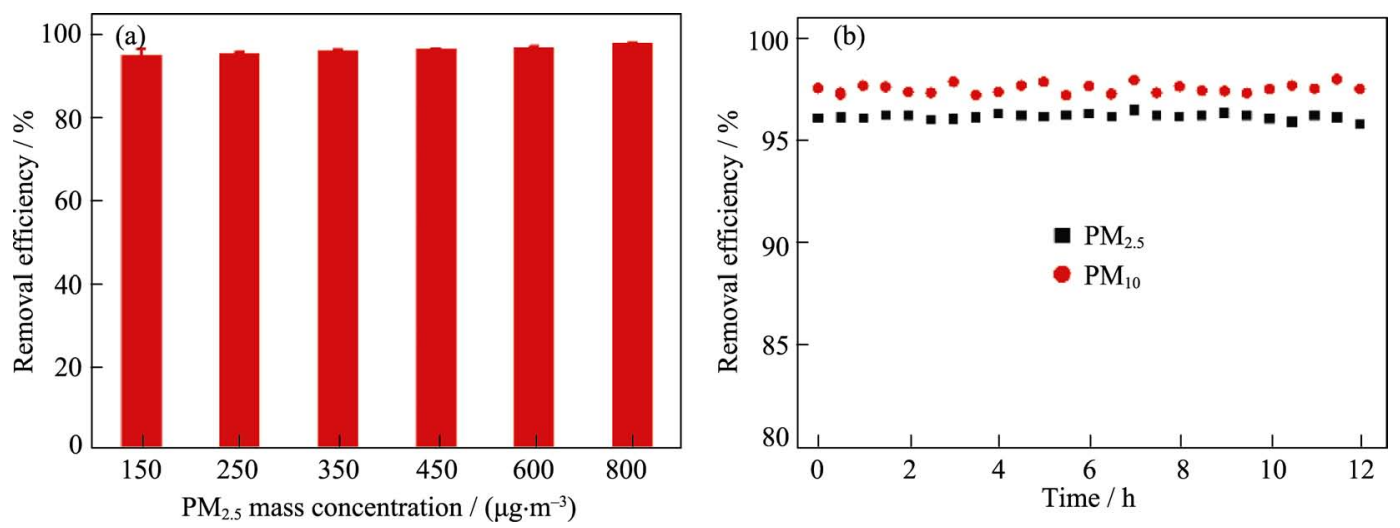

图 10 研制的新型羟基磷灰石超长纳米线基过滤纸在各种不同程度的污染环境中对空气 $\mathrm{PM}_{2.5}$ 细颗粒物的 过滤效率均高于 $95 \%$, 而且可以多次重复和长时间使用 ${ }^{[54]}$

Fig. $10 \quad \mathrm{PM}_{2.5}$ removal efficiencies of the as-prepared new kind of HAP nanowire-based filtration paper ${ }^{[54]}$

书籍、重要文件及档案的长久安全保存, 具有良好 的应用前景。此外, 该新型无机耐火纸也具有其它 多种用途。通过对 HAP 超长纳米线耐火纸进行功能 化, 可以制备出具有多种功能和不同用途的 HAP 超 长纳米线基新型纸张, 例如, 抗菌耐火纸、防水耐火 纸、荧光耐火纸、耐高温标签纸、光(电)缆用耐火 纸阻燃耐火包带、快速检测试纸、生物医用纸、高 效过滤纸等。这些功能化的耐火纸可以进一步拓展 HAP 超长纳米线基耐火纸的应用领域。新型 HAP 超长纳米线基耐火纸在特种纸、吸附过滤、生物医 学、隔热、耐高温、环境保护、能源等多个领域展 现出广阔的应用前景。

在今后的研究中, 需要进一步扩展新型 HAP 超 长纳米线基耐火纸的功能和应用领域, 实现新型无 机耐火纸的多功能化。未来的一个重要目标是实现 新型 HAP 超长纳米线基耐火纸的产业化和应用。要 实现这一目标, 目前遇到的瓶颈问题是作为新型无 机耐火纸制造原料的 HAP 超长纳米线的低成本和 批量化合成技术。目前, 已经在实验室实现了体积 为 $10 \mathrm{~L}$ 级反应釜的制备技术, 正在探索体积为 $100 \mathrm{~L}$ 级反应釜的制备技术。未来, 将发展 HAP 超长纳米 线的更大规模的批量化制备技术，还要进一步降低 制备成本, 以便使新型 HAP 超长纳米线基耐火纸向 产业化和实际应用方向推进。

\section{参考文献:}

[1] 法国国家图书馆遭遇水灾 一万余部书籍被淹[EB/OL]. [201710-25]. http://news.china.com.cn/world/2014-01/17/content_31222591. htm.

[2]俄图书馆失火百万史料焚毁 [EB/OL]. [2017-10-25]. http:// newspaper.jfdaily.com/jfrb/html/2015-02/02/content_63727.htm.

[3] CHEN F, ZHU Y J. Multifunctional calcium phosphate nanostructured materials and biomedical applications. Current Nanoscience, 2014, 10(4): 465-485.
[4] HUI J F, WANG X. Hydroxyapatite nanocrystals: colloidal chemistry, assembly and their biological applications. Inorganic Chemistry Frontiers, 2014, 1(3): 215-225.

[5] ŠUPOVÁ M. Substituted hydroxyapatites for biomedical applications: a review. Ceramics International, 2015, 41(8): 9203-9231.

[6] HAIDER A, HAIDER S, HAN S S, et al. Recent advances in the synthesis, functionalization and biomedical applications of hydroxyapatite: a review. RSC Advances, 2017, 7(13): 7442-7458.

[7] CHEN F, HUANG P, ZHU Y J, et al. The photoluminescence, drug delivery and imaging properties of multifunctional $\mathrm{Eu}^{3+} / \mathrm{Gd}^{3+}$ dual-doped hydroxyapatite nanorods. Biomaterials, 2011, 32(34): 9031-9039.

[8] LIU C S, HUANG Y, CUI J H. Kinetics of hydroxyapatite precipitation at $\mathrm{pH} 10$ to 11. Biomaterials, 2001, 22(4): 301-306.

[9] ZHANG C M, LI C X, HUANG S S, et al. Self-activated luminescent and mesoporous strontium hydroxyapatite nanorods for drug delivery. Biomaterials, 2010, 31(12): 3374 -3383.

[10] ZHU Y J, CHEN F. Microwave-assisted synthesis of calcium phosphate nanostructured materials in liquid phase. Progress in Chemistry, 2015, 27(5): 459-471.

[11] CHENG Y, WANG M, WANG X X, et al. Investigation on in vitro osteogenic properties of multiple-doped hydroxyapatite with natural bone content. Journal of Inorganic Materials, 2016, 31(12): 1341-1346.

[12] LU B Q, ZHU Y J, CHEN F. Highly flexible and noninflammable inorganic hydroxyapatite paper. Chemistry-A European Journal, 2014, 20(5): 1242-1246.

[13] KANDORI K, KURODA T, TOGASHI S, et al. Preparation of calcium hydroxyapatite nanoparticles using microreactor and their characteristics of protein adsorption. Journal of Physical Chemistry B, 2011, 115(4): 653-659.

[14] ANWAR A, REHMAN I U, DARR J A. Low-temperature synthesis and surface modification of high surface area calcium hydroxyapatite nanorods incorporating organofunctionalized surfaces. Journal of Physical Chemistry C, 2016, 120(51): 29069-29076.

[15] CHEN H F, SUN K, TANG Z Y, et al. Synthesis of fluorapatite nanorods and nanowires by direct precipitation from solution. Crystal Growth \& Design, 2006, 6(6): 1504-1508.

[16] LV B Y, ZHAO L S, PU Y, et al. Facile preparation of controllableaspect-ratio hydroxyapatite nanorods with high-gravity technology for bone tissue engineering. Industrial \& Engineering Chemistry Research, 2017, 56(11): 2976-2983.

[17] ZHAO X Y, ZHU Y J, LU B Q, et al. Hydrothermal synthesis of hydroxyapatite nanorods using pyridoxal-5'-phosphate as a phos- 
phorus source. Materials Research Bulletin, 2014, 55: 67-70.

[18] MA M G, ZHU Y J, CHANG J. Monetite formed in mixed solvents of water and ethylene glycol and its transformation to hydroxyapatite. Journal of Physical Chemistry B, 2006, 110(29): 14226-14230.

[19] ZHAO X Y, ZHU Y J, QI C, et al. Hierarchical hollow hydroxyapatite microspheres: microwave-assisted rapid synthesis by using pyridoxal-5'-phosphate as a phosphorus source and application in drug delivery. Chemistry-An Asian Journal, 2013, 8(6): $1313-1320$

[20] ZHAO J, ZHU Y J, CHENG G F, et al. Microwave-assisted hydrothermal rapid synthesis of amorphous calcium phosphate nanoparticles and hydroxyapatite microspheres using cytidine 5'-triphosphate disodium salt as a phosphate source. Materials Letters, 2014, 124: 208-211.

[21] PARK S Y, KIM K I, PARK S P, et al. Aspartic acid-assisted synthesis of multifunctional strontium-substituted hydroxyapatite microspheres. Crystal Growth \& Design, 2016, 16(8): 4318-4326.

[22] CHEN F, ZHU Y J, WANG K W, et al. Surfactant-free solvothermal synthesis of hydroxyapatite nanowire/nanotube ordered arrays with biomimetic structures. CrystEngComm, 2011, 13(6): 1858-1863.

[23] ZHAO X Y, ZHU Y J, CHEN F, et al. Hydrothermal synthesis of hydroxyapatite nanorods and nanowires using riboflavin-5'phosphate monosodium salt as a new phosphorus source and their application in protein adsorption. CrystEngComm, 2013, 15(39): 7926-7935.

[24] BRAMHE S, KIM T N, BALAKRISHNAN A, et al. Conversion from biowaste venerupis clam shells to hydroxyapatite nanowires. Materials Letters, 2014, 135: 195-198.

[25] AI M, DU Z Y, ZHU S Q, et al. Composite resin reinforced with silver nanoparticles-laden hydroxyapatite nanowires for dental application. Dental Materials, 2017, 33(1): 12-22.

[26] HE J Y, ZHANG K S, WU S B, et al. Performance of novel hydroxyapatite nanowires in treatment of fluoride contaminated water. Journal of Hazardous Materials, 2016, 303(13): 119-130.

[27] JIANG Y Y, ZHU Y J, CHEN F, et al. Solvothermal synthesis of submillimeter ultralong hydroxyapatite nanowires using a calcium oleate precursor in a series of monohydroxy alcohols. Ceramics International, 2015, 41(4): 6098-6102.

[28] ZHANG Y G, ZHU Y J, CHEN F, et al. Ultralong hydroxyapatite nanowires synthesized by solvothermal treatment using a series of phosphate sodium salts. Materials Letters, 2015, 144: 135-137.

[29] LI H, ZHU Y J, JIANG Y Y, et al. Hierarchical assembly of monodisperse hydroxyapatite nanowires and construction of high-strength fire-resistant inorganic paper with high-temperature flexibility. ChemNanoMat, 2017, 3(4): 259-268.

[30] QI C, TANG Q L, ZHU Y J, et al. Microwave-assisted hydrothermal rapid synthesis of hydroxyapatite nanowires using adenosine 5'-triphosphate disodium salt as phosphorus source. Materials Letters, 2012, 85: 71-73.

[31] LIN K L, LIU X G, CHANG J, et al. Facile synthesis of hydroxyapatite nanoparticles, nanowires and hollow nano-structured microspheres using similar structured hard-precursors. Nanoscale, 2011, 3(8): 3052-3055.

[32] YANG Z, HUANG Y, CHEN S T, et al. Template synthesis of highly ordered hydroxyapatite nanowire arrays. Journal of Materials Science, 2005, 40(5): 1121-1125.

[33] COSTA D O, DIXON S J, RIZKALLA A S. One- and three-dimensional growth of hydroxyapatite nanowires during sol-gel-hydrothermal synthesis. ACS Applied Materials \& Interfaces, 2012, 4(3): 1490-1499.

[34] CAO M H, WANG Y H, GUO C X, et al. Preparation of ultrahigh-aspect-ratio hydroxyapatite nanofibers in reverse micelles under hydrothermal conditions. Langmuir, 2004, 20(11): 4784-4786.

[35] CHEN F, ZHU Y J. Large-scale automated production of highly ordered ultralong hydroxyapatite nanowires and construction of various fire-resistant flexible ordered architectures. ACS Nano, 2016, 10(12), 11483-11495.

[36] VORONOV R S, PAPAVASSILIOU D V, LEE L L. Review of fluid slip over superhydrophobic surfaces and its dependence on the contact angle. Industrial \& Engineering Chemistry Research, 2008, 47(8): 2455-2477.

[37] DYETT B P, WU A H, LAMB R N. Mechanical stability of surface architecture-consequences for superhydrophobicity. ACS Applied Materilas \& Interfaces, 2014, 6(21): 18380-18394.

[38] CHEN F F, ZHU Y J, XIONG Z C, et al. Highly flexible superhydrophobic and fire-resistant layered inorganic paper. ACS Applied Materials \& Interfaces, 2016, 8(50): 34715-34724.

[39] CHEN F F, ZHU Y J, XIONG Z C, et al. Inorganic nanowires-assembled layered paper as the valve for controlling water transportation. ACS Applied Materials \& Interfaces, 2017, 9(12): 11045-11053.

[40] XIONG Z C, ZHU Y J, CHEN F F, et al. One-step synthesis of silver nanoparticle-decorated hydroxyapatite nanowires for the construction of highly flexible free-standing paper with high antibacterial activity. Chemistry-A European Journal, 2016, 22(32): 11224-11231.

[41] XIONG Z C, YANG Z Y, ZHU Y J, et al. Ultralong hydroxyapatite nanowires-based paper co-loaded with silver nanoparticles and antibiotic for long-term antibacterial benefit. ACS Applied Materials \& Interfaces, 2017, 9(27): 22212-22222.

[42] DONG L Y, ZHU Y J. A new kind of fireproof, flexible, inorganic, nanocomposite paper and its application to the protection layer in flame-retardant fiber-optic cables. Chemistry-A European Journal, 2017, 23(19): 4597-4604.

[43] CHEN F F, ZHU Y J, XIONG Z C, et al. Hydroxyapatite nanowires@metal-organic framework core/shell nanofibers: templated synthesis, peroxidase-like activity, and derived flexible recyclable test paper. Chemistry-A European Journal, 2017, 23(14): 3328-3337.

[44] SUN T W, ZHU Y J, CHEN F. Highly flexible multifunctional biopaper comprising chitosan reinforced by ultralong hydroxyapatite nanowires. Chemistry-A European Journal, 2017, 23(16): 3850-3862.

[45] SUN T W, ZHU Y J, CHEN F, et al. Ultralong hydroxyapatite nanowire/collagen biopaper with high flexibility, improved mechanical properties and excellent cellular attachment. Chemistry-An Asian Journal, 2017, 12(6): 655-664.

[46] XIE Y F, HE W M, LI F, et al. Luminescence enhanced $\mathrm{Eu}^{3+} / \mathrm{Gd}^{3+}$ co-doped hydroxyapatite nanocrystals as imaging agents in vitro and in vivo. ACS Applied Materials \& Interfaces, 2016, 8(16): 10212-10219.

[47] ZHENG X Y, LIU M Y, HUI J F, et al. $\mathrm{Ln}^{3+}$-doped hydroxyapatite nanocrystals: controllable synthesis and cell imaging. Physical Chemistry Chemical Physics, 2015, 17(31): 20301-20307.

[48] PERERA T S H, HAN Y C, LU X F, et al. Rare earth doped apatite nanomaterials for biological application. Journal of Nanomaterials, 2015: 705390

[49] SUN Y X, YANG H, TAO D L. Preparation and characterization of $\mathrm{Eu}^{3+}$-doped fluorapatite nanoparticles by a hydrothermal method. Ceramics International, 2012, 38(8): 6937-6941.

[50] TESCH A, WENISCH C, HERRMANN K H, et al. Luminomagnetic $\mathrm{Eu}^{3+}$ and $\mathrm{Dy}^{3+}$-doped hydroxyapatite for multimodal imaging. 
Materials Science \& Engineering C, 2017, 81: 422-431.

[51] YANG R L, ZHU Y J, CHEN F F, et al. Luminescent, fire-resistant and water-proof ultralong hydroxyapatite nanowires-based paper for multimode anti-counterfeiting application. ACS Applied Materials \& Interfaces, 2017, 9(30): 25455-25464.

[52] TAO J, ZHANG L M, CAO J J, et al. A review of current knowledge concerning PM2.5 chemical composition, aerosol optical properties and their relationships across china. Atmospheric Chem- istry and Physics, 2017, 17(15): 9485-9518.

[53] BO M, SALIZZONI P, CLERICO M, et al. Assessment of indoor-outdoor particulate matter air pollution: a review. Atmosphere, 2017, 8(8): Article Number 136.

[54] XIONG Z C, YANG R L, ZHU Y J, et al. Flexible hydroxyapatite ultralong nanowires-based paper for highly efficient and multifunctional air filtration. Journal of Materials Chemistry A, 2017, 5(33): 17482-17491.

\section{我与严东生先生}

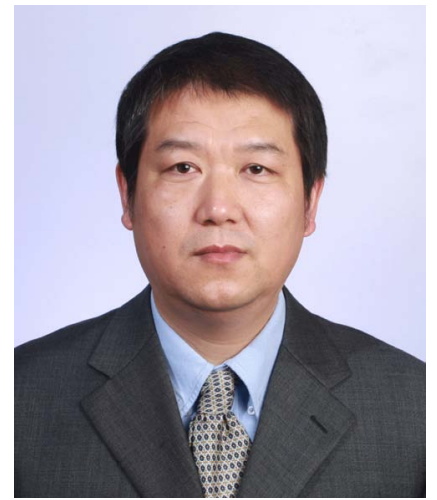

我是 2002 年入选中科院“百人计划”从美国回国到中国科学院上海硅酸盐 所工作的。初到单位, 没有实验室和研究生, 白手起家, 首先面临的一个问题 就是要确定研究方向。严先生给予我很多关心、支持和帮助, 很热情地与我面 谈, 了解我的情况, 并在学术上给予我很多非常宝贵的指导。在此后的十多年 里, 严先生多次与我面谈, 了解我的科研进展, 我发表的许多论文也打印出来 请严先生审阅, 严先生非常认真地与我讨论论文中的科学问题, 并对我的科 研工作给予了很多鼓励和指导。严先生的谆谆教诲对我的研究工作起到了非 常重要的指引作用, 此后我的研究工作取得了较好进展, 2002 年成为研究员 和博士生导师; 2004 年在德国“应用化学”上发表的论文已被引用超过 460 次; 2005 年获得“百人计划”项目终期评估“优秀”; 2007 年入选上海市“优秀学科带 头人”; 多次获得优秀导师奖。回想我在上海硅酸盐研究所的科研工作经历, 我的内心充满了对严先生的无 限怀念和感激之情。严先生慈祥的音容笑貌和孜孜不倦追求科学的精神永远铭记在我的心中。 